\title{
Distinctive Motor Stereotypy in a 4-month-old Infant with Congenital CMV Infection
}

\author{
Dawood Tafti ${ }^{1}$, David Ahn ${ }^{2}$ \\ ${ }^{1}$ Department of Radiology, San Antonio Military Medical Center, 3551 Roger Brooke Dr. JBSA-Fort Sam Houston, TX, United \\ States. 78234; ${ }^{2}$ Department of Anesthesiology, Walter Reed National Military Medical Center, 104 Wood Rd, Bethesda, MD, \\ United States. 20814.
}

\section{Corresponding Author:}

Dr. Dawood Tafti

Email: dtafti121@gmail.com

This is an Open Access article distributed under the terms of the Creative Commons Attribution License (creativecommons.org/ licenses/by/3.0).

\section{Received : July 11,2020}

Accepted : November 13, 2020

Published : December 30, 2020

\begin{abstract}
Background: Abnormal stereotypy, although described in non-human primates with congenital cytomegalovirus infection, has not been described in human infants and neonates with central nervous system cytomegalovirus disease. Case Report: We present a case of a 4-month-old infant male with congenital cytomegalovirus infection who presented for complaints of repetitive and conscious stereotypy of the upper and lower extremities for the majority of the patient's waking period. Conclusion: Asymptomatic neonates with central nervous system cytomegalovirus infection may go on to develop permanent neurologic sequelae of disease. The early recognition of congenital cytomegalovirus disease is critical to help initiate early treatment interventions. Clinicians should maintain a low threshold for suspicion for TORCH infections and neurologic disorders in infants with an abnormal stereotypy.
\end{abstract}

Keywords: Central Nervous System Infections, Cytomegalovirus, Infant, Muscle Hypotonia.

\section{Introduction}

Congenital cytomegalovirus (CMV) infection remains the principal cause of brain damage in children, as well as being the most common congenital infection in the United States [1,2]. An estimated 40,000 newborns are born with CMV annually in the United States, affecting approximately $1 \%$ of all live births [3]. An extensive number of studies have been undertaken to characterize the symptomatic presentation of congenital CMV disease, and various manifestations of central nervous system (CNS) disease have been described in CMV infection [4,5]. Although $50-90 \%$ of patients with symptomatic congenital CMV infection will develop long term deficits such as mental retardation or other cognitive defects, there is a substantial percentage of asymptomatic patients (approximately 7-25\%) who go on to develop deficits later in life [6-8].

Some studies suggest asymptomatic CMV disease may be associated with a broad range of subtle neurodevelopmental sequelae [9]. This has brought on the need for close identification and follow-up of infants with subtle neurologic symptoms who have otherwise normal physical exams. Careful assessment of development and subtle physical exam findings are critical to identify those who require early interventions for corrective measures [10]. We present a rare case of a 4-month-old male with congenital CMV infection who demonstrated an abnormal repetitive and conscious motor stereotypy as the earliest clinical sign of disease. To the best of our knowledge, an abnormal stereotypy in an infant has not been described as a potential manifestation of intracranial CNS CMV disease. 


\section{Case Report}

Our case involved a 4-month-old otherwise asymptomatic male infant with congenital CMV diagnosed by urine and blood PCR soon after birth. The patient was found to have intracranial calcifications on head ultrasound at an outside facility. The mother of the patient noted a 2-month history of a distinctive and persistent stereotypy involving high velocity and high amplitude synchronized flexion and extension of the lower extremities. The mother of the patient stated that she swaddled the patient for most of the day as this effectively suppressed the stereotypy. These movements on the mother's estimation occurred "more than $90 \%$ " of the patient's waking period if the patient was not swaddled to suppress the stereotypy.

Physical exam revealed a well-appearing infant with findings of mild diffuse hypotonia of the upper extremities, hypertonia of the lower extremities, and the above-described stereotypy during the entire duration of the approximately 35-minute clinic visit. Of note, this stereotypy did resolve with the momentary clasping of the feet by the provider during the physical exam and on placing the infant prone with a subsequent nearimmediate resumption of the stereotypy on placing the infant back to supine position. The infant was in no apparent distress and was appropriately tracking objects with his eyes during the stereotypy. Findings were not consistent with seizure-like activity and EEG was deferred. A subsequent MRI study was ordered and demonstrated T2 hyperintense signal along the periventricular white matter consistent with CMV infection [Fig.1].

\section{Discussion}

The pediatric neurologic exam is an important cornerstone of the well-child visit. Components of the exam include evaluation of the spine for signs of underlying malformation such as tufts of hair and dimples. The pediatric neurologic exam also includes evaluating head size and shape to evaluate for findings such as microcephaly and

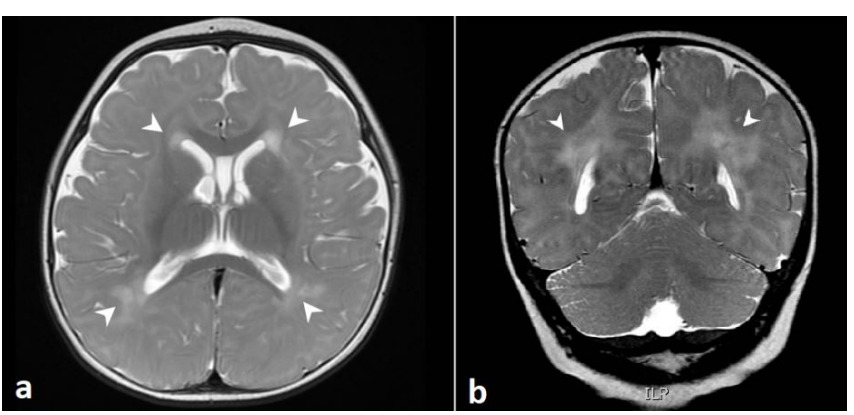

Fig.1: 4-month-old male infant with congenital CMV; (a) Single axial MRI slice of the brain at the level of the lateral ventricles demonstrates high T2 white matter changes in a periventricular distribution; (b) Single coronal MRI slice of the brain at the level of the occipital horns of the lateral ventricles demonstrate high $T 2$ white matter changes in a periventricular distribution.

macrocephaly. The evaluation of the cranial nerves and the reflexes of an infant also allows the provider to assess for appropriate neurologic development during a well-child visit. Finally, evaluation of motor activity primarily involves the assessment of normal spontaneous activity and assessing for deficits in movement.

A motor stereotypy is defined broadly as an involuntary and coordinated repetitive movement that is purposeless [11]. A complex motor stereotypy includes stereotypies that involve the arms and sometimes legs. Stereotypies are often rhythmic and last for seconds to minutes. They can occur in clusters and can be suppressed by distracting a patient or having the patient initiate another action [12]. With the exception of normal stereotypies such as thumb sucking, unique and abnormal stereotypies in infants are rare. Although stereotypies are poorly understood, there has been greater attention paid to stereotypies developing in early childhood due to their presentation in patients affected by autistic spectrum disorder [13].

Although stereotypies have been described in Rhesus macaque primates with CMV disease, multiple studies describing the range of symptomatic manifestations of CMV disease in humans have not listed abnormal stereotypies 
as a manifestation of congenital CMV disease in human infants and neonates [5,14-17]. Continuing studies have shown that approximately $13 \%$ of asymptomatic neonates may go on to develop permanent sequelae making the early recognition of congenital CMV disease in infants important to initiate early interventions [18].

Early interventions applied to infants affected with intracranial CMV infection are known to improve outcomes, without which neurological outcomes worsen $[19,20]$. A significant proportion of infants with CMV disease have subtle neurologic findings. Infections can also be clinically silent. Stereotypies are a poorly understood clinical entity, especially in the field of pediatric neurology.

\section{Conclusion}

We present our case as an opportunity to add to the list of neurologic manifestations of intracranial CMV disease. This case also illustrates the importance of assessing for normal motor activity during the neurologic assessment of the well-child visit.

Contributors: DT: manuscript writing, and patient management; DA: reviewing the literature, manuscript editing and patient management. DT will act as a study guarantor. Both authors approved the final version of this manuscript and are responsible for all aspects of the study.

Funding: None; Competing interests: None stated.

\section{References}

1. Nelson CT, Demmler GJ. Cytomegalovirus infection in the pregnant mother, fetus, and newborn infant. Clin Perinatol. 1997;24:151-160.

2. Boppana SB, Fowler KB, Britt WJ, Stagno S, Pass RF. Symptomatic congenital cytomegalovirus infection in infants born to mothers with preexisting immunity to cytomegalovirus. Pediatrics. 1999;104(1 Pt 1):55-60.

3. Noyola DE, Demmler GJ, Nelson CT, Griesser C, Williamson WD, Atkins JT, et al. Early predictors of neurodevelopmental outcome in symptomatic congenital cytomegalovirus infection. J Pediatr. 2001;138(3):325-331.

4. Demmler GJ. Congenital cytomegalovirus infection and disease. Adv Pediatr Infect Dis. 1996;11:135-162.

5. Boppana SB, Pass RF, Britt WJ, Stagno S, Alford CA. Symptomatic congenital cytomegalovirus infection: neonatal morbidity and mortality. Pediatr Infect Dis J. 1992;11(2):93-99.

6. Bale JF. Human cytomegalovirus infection and disorders of the nervous system. Arch Neurol. 1984;41:310-320.

7. Arav-boger R, Pass RF. Diagnosis and management of cytomegalovirus infection in the newborn. Pediatr Ann. 2002;31(11):719-725.

8. Stagno S, Britt W. Cytomegalovirus infections. In: Remington JS, Klein JO, Wilson CB, Baker CJ, editors. Infectious diseases of the fetus and newborn infant. $6^{\text {th }}$ ed. Philadelphia: Saunders. 2006:739-781.

9. Baker CJ, eds. Infectious Diseases of the Fetus and Newborn Infant, $6^{\text {th }}$ ed., Philadelphia:Elsevier Saunders, 2006;739-772.

10. Williamson WD, Percy AK, Yow MD, Gerson P, Catlin FI, Koppelman ML. Asymptomatic congenital cytomegalovirus infection: Audiologic, neuroradiologic, and neurodevelopmental abnormalities during the first year. Am J Dis Child. 1990;144(12):1365-1368.

11. Jankovic J. Stereotypies. In: Marsden CD, Fahn S, editors. Movement disorders. $3^{\text {rd }}$ ed. London: Butterworth Heinemann; 1994. pp. 501-517.

12. LaGrow SJ, Repp AC. Stereotypic responding: a review of intervention research. Am J Ment Defic. 1984;88:595609.

13. Muthugovindan D, Singer H. Motor stereotypy disorders. Curr Opin Neurol. 2009;22:131-136.

14. Sean PE. Herpes virus. In: Barton L, Friedman N (eds). The Neurological Manifestations of Pediatric Infectious Diseases and Immunodeficiency Syndromes. Humana Press. 2008:pp. 3-40.

15. Clemmons EA, Gumber S, Strobert E, Bloomsmith MA, Jean SM. Self-injurious behavior secondary to cytomegalovirus-induced neuropathy in an SIV-infected Rhesus Macaque (Macaca mulatta). Comp Med. 2015;65(3):266-270.

16. Kylat RI, Kelly EN, Ford-jones EL. Clinical findings and adverse outcome in neonates with symptomatic congenital cytomegalovirus (SCCMV) infection. Eur J Pediatr. 2006;165(11):773-778.

17. Yamamoto AY, Mussi-pinhata MM, Cristina P, Pinto G, Moraes figueiredo LT, Jorge SM. Congenital cytomegalovirus infection in preterm and full-term newborn infants from a population with a high seroprevalence rate. Pediatr Infect Dis J. 2001;20(2):188192.

18. Istas AS, Demmler GJ, Dobbins JG, Stewart JA. Surveillance for congenital cytomegalovirus disease: a report from the National Congenital Cytomegalovirus Disease Registry. Clin Infect Dis. 1995;20(3):665-670.

19. Dollard SC, Grosse SD, Ross DS. New estimates of the prevalence of neurological and sensory sequelae and mortality associated with congenital cytomegalovirus infection. Rev Med Virol. 2007;17(5):355-363.

20. Ciorba A, Bovo R, Trevisi P, Bianchini C, Arboretti R, Martini A. Rehabilitation and outcome of severe profound deafness in a group of 16 infants affected by congenital cytomegalovirus infection. Eur Arch Otorhinolaryngol. 2009;266:1539-1546. 